\title{
REAL-TIME SUPPORT FOR ONLINE CONTROLLER SUPERVISION AND OPTIMISATION
}

\author{
Markus Deppe, Oliver Oberschelp \\ Mechatronics Laboratory Paderborn ${ }^{1}$ \\ University of Paderborn \\ Germany
}

\begin{abstract}
This paper presents three methods for the supervision and optimisation of a controller by means of an exemplary application. The supervision is used for error recognition, analysis, and processing. The basic principle comprises the use of different controllers. $A$ robust controller as a fail-safe device and a controller to be tested are implemented. In the case of an error during the tests the system automatically switches to the robust controller. This mechanism is implemented by means of a finitestate machine. A precision supervision compares setpoints and measured values. The difference is weighted and transformed into discrete events which affect the switching between the different controllers. Through a spectrum analysis appropriate for realtime use, supervision is effected in the frequency domain. The results of the computation can be used directly for online optimisation. It is shown that in the case of real-time, a synchronous computation of the frequency spectrum is more useful than an asynchronous one. The results are presented with a current application from the domain of railway technology. This is a suspension/tilt-module testbed employed with the research project "Neue Bahntechnik Paderborn".
\end{abstract}

\section{Introduction}

Ever increasing demands on the complexity, features, and possibilities linked with mechatronic systems (e.g., operational range, security, comfort, and costs) can no longer be met just by using simple adaptive controls.

The potential inherent in novel circuit techniques and ever more potent microprocessors, however, opens up a new dimension for online optimization and supervision that will exceed the conventional view of the adaptability (Isermann, Lachmann, Matko 1992) of a system (esp. in the domain of adaptive controls).

${ }^{1}$ MLaP, Pohlweg 98, D-33098 Paderborn, http://www.MLaP.de

The original version of this chapter was revised: The copyright line was incorrect. This has been corrected. The Erratum to this chapter is available at DOI: 10.1007/978-0-387-35409-5_23 
The tasks relating to online supervision and optimisation can generally be structured as follows:

Supervision of the hardware, e.g., linking of the sensors and the state of the processor.

Improvement of the controller function by an optimisation.

Supervision of the software to ensure controller stability and precision.

HiL testbeds to test mechatronic components are mechatronic systems as well. Here manual or automated tests and optimisations are performed. In most cases the level of the electrical, the mechanical and the hydraulic power during the tests and/or the optimisations is quite high. The risk of damage for man or machine has to be minimised as far as possible. Switching off the energy supply is not the best solution in every possible case. An automated fail-safe reaction provided by the information processing is the better alternative in comparison with (slow) manual interactions. That is why the testbed has to be equipped to ensure safety even in cases of operating errors caused by the user or software. These experiences are our motivation for the work presented.

We are going to demonstrate a controller switching (from test to robust and back), a controller precision supervision, and finally a spectral-resonance supervision for a HiL testbed.

\section{The Design of Mechatronic Systems}

At the Mechatronics Laboratory Paderborn (MLaP) we developed the software environment CAMeL (Computer-Aided Mechatronics Laboratory) (Meier-Noe, Hahn, 1999). It allows to do studies of the dynamical behaviour that precede and then accompany the constructive design (Lückel, Toepper, et al., 1999).

All components and their arrangement are represented in the computer by means of graphical support. Every component of the entire system is described in its discipline-specific manner. Thus a thorough description of rigid bodies as elements of the mechanical supporting structure, e.g., requires information on the respective mass, inertia tensor and position of the points relating to the coupling, application of force, and measurement. In a subsequent step this structural representation of the mechatronic system by its different discipline-specific descriptions is made uniform in a mathematical-symbolical representation. All basic elements are transformed into the explicit state-space representation and the subsystems are coupled by their input/output relations. Then this mathematical model is transformed into a numerical one that the computer can process and evaluate. As a result the behaviour of motion is computed, yielding information on time- and frequency behaviour, stability and sensibility and indicating if the system does or does not function properly. Now, in addition to the geometrical dimensions, other essential physical variables are available to the developer, e.g., forces and torques arising in the system, pressures, mechanical and electrical tensions. Moreover, the stiffness of the system and forms of self-oscillation can be analyzed. 


\section{The Structure of Hybrid Information Processing}

To supervise or optimise the controllers a discrete component we call operator is assigned to each continuous controller.

This newly introduced entity between controller and operator is named OperatorController Module (Naumann, 2000). Figure 1 shows the basic structure of an Operator-Controller Module. The operator retrieves information from the controller and updates the latter if necessary. The update may be limited to simple parameter tuning but can even imply switching of the controller structures:

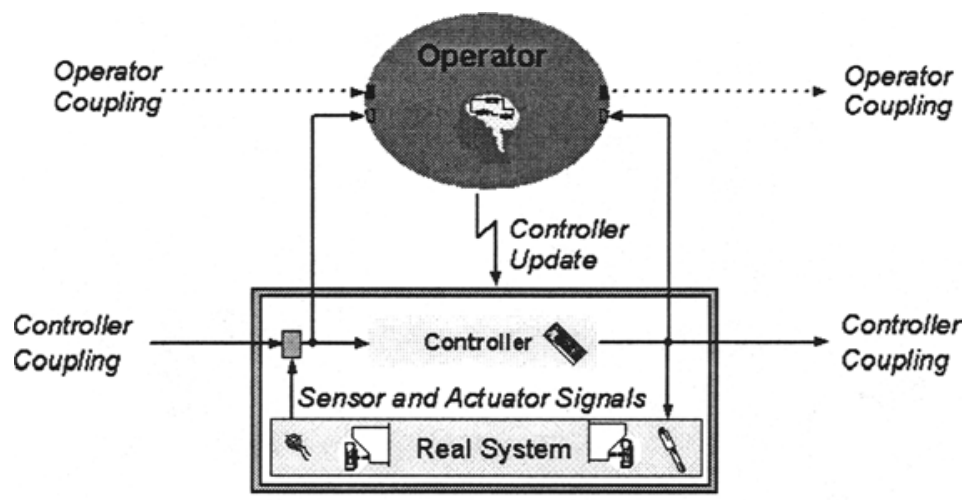

Mechatronic System (MFM, AMS, CMS)

Figure 1. The Operator-Controller Module.

In this way a hybrid system in the sense of a mixed discrete/continuous system comes into existence. But this simple subdivision into discrete and continuous parts hardly resembles a powerful concept for the design of complex mechatronic systems. Thus, combining the operator-controller module with a modular and hierarchical structuring of mechatronic systems is the next step to take (Honekamp, et al., 1997).

At the lowest level of complex systems we defined the Mechatronic Function Modules (MFM). A Mechatronic Function Module consists of a passive mechanical frame, sensors, actuators, and hybrid information processing. The MFM can make up a hierarchical structure.

The next level of hierarchy is that of the Autonomous Mechatronic System (AMS). An AMS consists of a passive mechanical frame, sensors, and information processing. It has no actuators of its own but uses the mechanically coupled MFMs for actuation. The highest hierarchical level is that of Crosslinked Mechatronic Systems, CMS in short. On this level several AMSs are linked together by a communication infrastructure to make up a co-operating system. Co-operating robots or automobiles are examples of CMS.

We have here a highly generalised structuring concept. In this paper we want to direct your attention to the supervision of a hardware-in-the-loop testbed for a railway suspension/tilt module. 


\section{Our Example: Testbed for a Railway Suspension/Tilt Module}

Within the project "Neue Bahntechnik Paderborn" (http://nbp-www.upb.de) a modular railway concept is currently elaborated that combines modern undercarriage technology with the advantages of the Transrapid and the use of existing railway tracks (Lückel, Grotstollen, et al., 1999).

Its actively controlled suspension/tilt module (Figure 2) realising the spring concept for the carriage body of the shuttles serves as an example. The carriage body supported by airsprings is damped and tilts by means of a hydraulic base displacement at the upper plate.

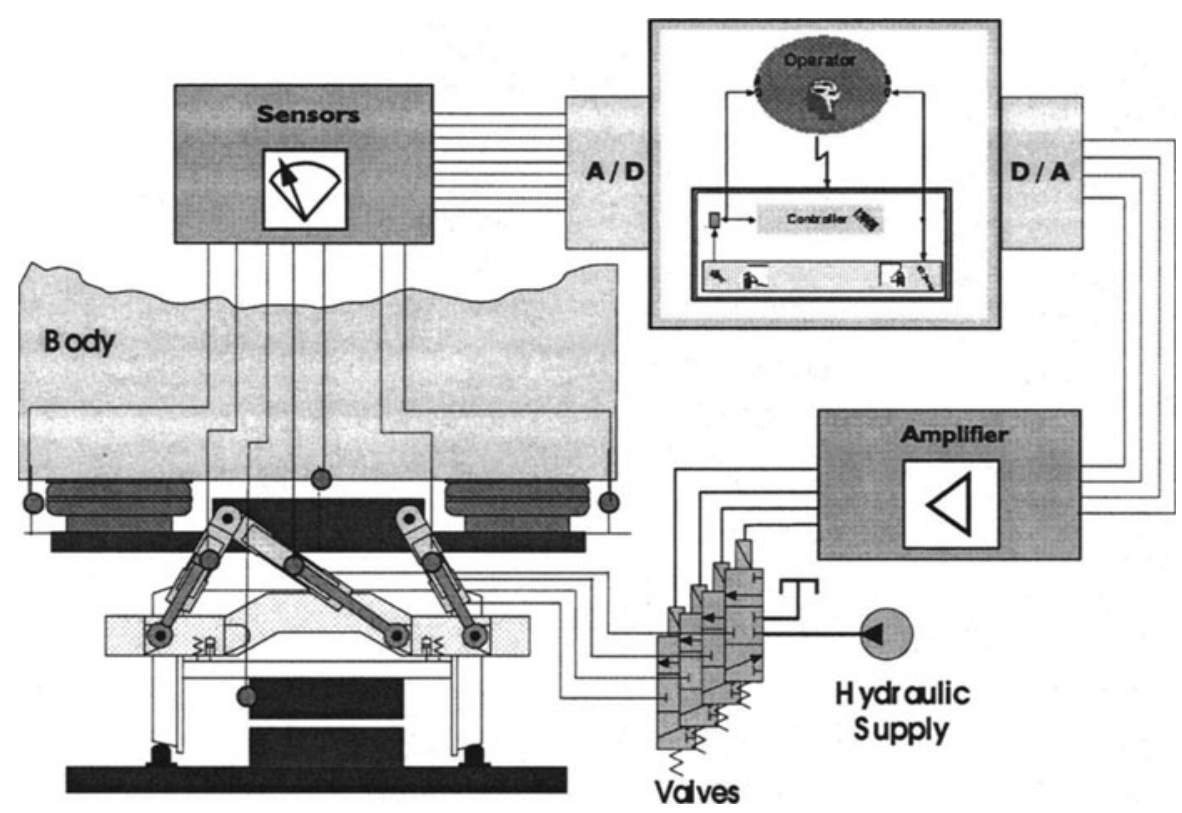

Figure 2. The railway suspension/tilt module.

So the main task of the controller is to provide active damping of the carriage body. This can be achieved by generating forces proportional to the relative velocity between body and upper plate by means of the actuators. The damping frequency range is limited to the bandwidth of the controller. The hydraulic valves are controlled by a hybrid information processing (Operator-Controller Modules) connected via sensors, amplifiers, and DA/AD converters.

To design this module the $M L a P$ disposes of a hardware-in-the-loop testbed (Lückel, Liu, et al., 2000). Our testbed includes additional hydraulic actuators to generate synthetic forces and displacements of the primary suspension. These actuators are not shown in Figure 2.

The testbed can be seen as an AMS (Figure 3), providing the mechanical, hydraulic and informational coupling of the underlying MFMs. These MFMs are the hydraulic power supply, the controlled testbed actuators, and the controlled 
suspension/tilt module itself. The AMS and each MFM contain their own OperatorController Module. In the following, we will refer to this structure to expound the implemented testbed operator and the assessment functions for controller switching.

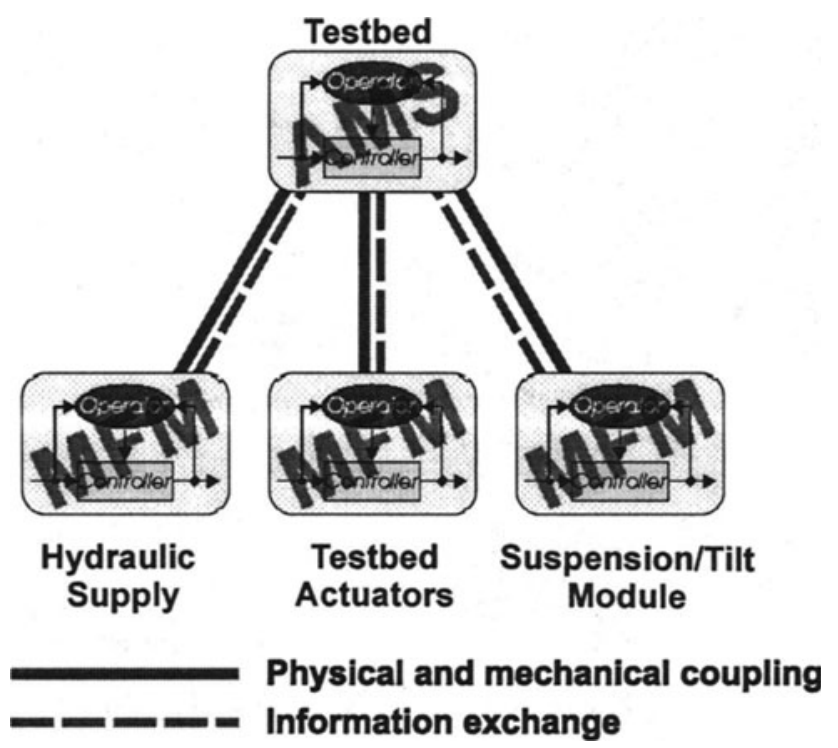

Figure 3. Hierarchical structure of the testbed.

\section{Real-time Testbed Supervision}

The testbed operator manages the different testbed states to provide a fail-safe and transparent interaction with the user of the testbed.

This component is located within the operator part on the AMS level of the system. For the testbed five states are defined (Figure 4). Within the initial state the hydraulic supply is switched off. This state can be reached from all other states when the turnOff event is triggered, except for the Fade state. In the Robust Control state the hydraulic supply is switched on while a robust controller is active. From there the user can switch to the Fade state in which the robust controller and the controller under test crossfade automatically.

In the Test Control state the system supervision can automatically decide to switch to the Test Failed state which means a return to robust control. The switching is effected by the local MFM operator. The automatic fail test is assessed synchronously within the local MFM controller section, resulting in a well-balanced computational load. With Test Failed the system can be switched off or a new test with altered test controller parameters can be started. 


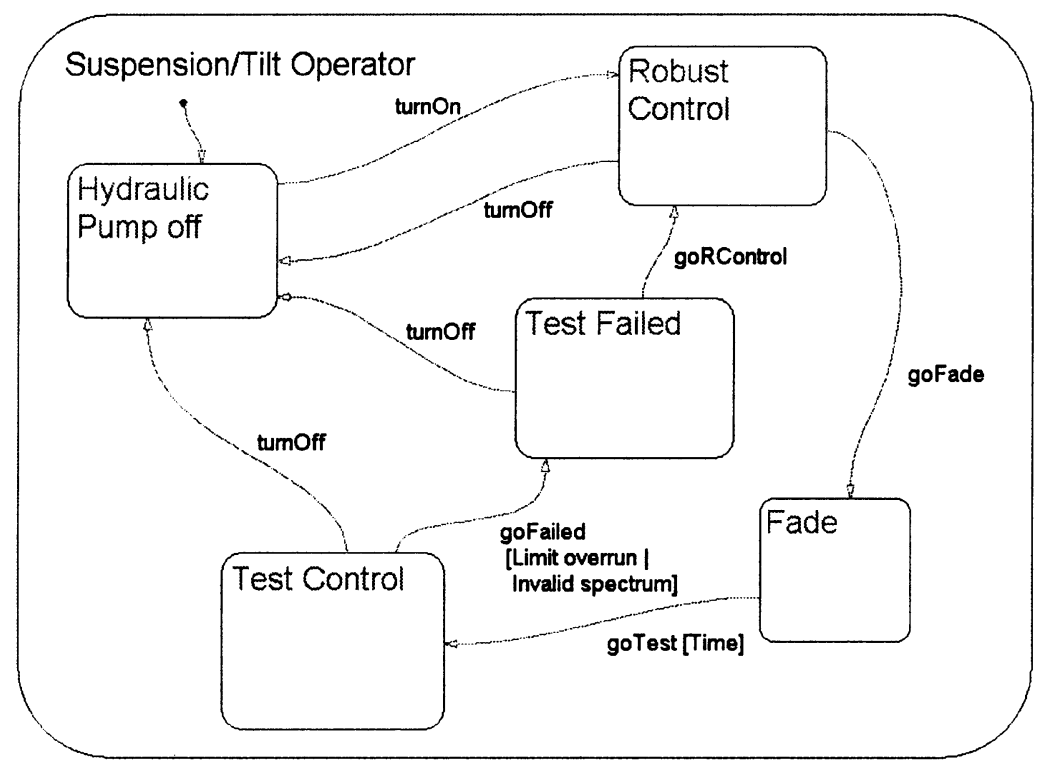

Figure 4. State chart of the discrete suspension/tilt testbed management.

\section{Real-time Actuator Precision Supervision}

To ensure safe operation it is very useful to monitor the precision of the hydraulic actuator control.

This task is located in the respective controller section both of the testbed MFM for actuation and of the suspension/tilt module MFM. It is implemented as a synchronous dataflow which compares the displacement of the actuator with that of a reference model (Figure 5).

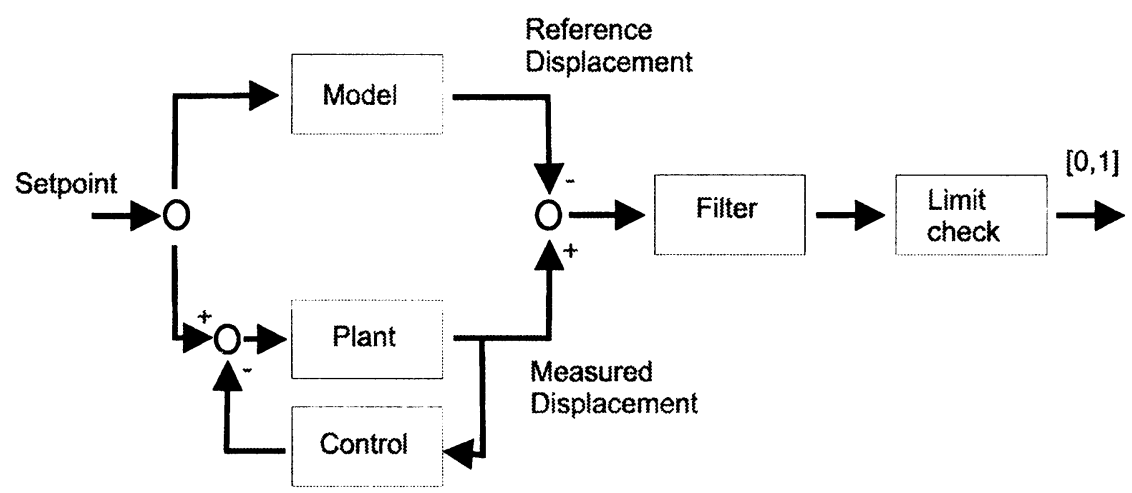

Figure 5. Dataflow of the actuator precision supervision. 
Absolute and relative limits can be provided to find out if the limit check succeeds or fails. The computed actuator deviation from the reference displacement can directly be used as a cost function for online optimisation. The suspension/tilt MFM and the testbed MFM operator sections comprise the components to switch between the robust controller and the controller under test.

\section{Real-time Spectral Evaluation}

To supervise the hydraulic valve sliders in the frequency domain we have done a real-time dataflow-based implementation of a discrete Fourier transformation (DFT) algorithm. This task is implemented in a synchronous manner (filter) and therefore placed in the suspension/tilt controller section.

Model-based reference signals are compared with measured signals by means of computing the least-squares cost function (Figure 6). The spectra comparison can directly be used as a cost function for online optimisation.

The implemented synchronous DFT requires a more complex multiplication $\left(O(N)=N^{2}\right)$ than an asynchronous one which would be able to massively exploit symmetries (Johnson, 1991). For the synchronous DFT filter only standard optimisations like the use of register variables and sine/cosine tables are possible. For real-time computation, however, there are two major advantages with the synchronous solution:

One gets results faster with a deterministic timing behaviour (immediately after each DFT sampling period).

The computational load will be well balanced because computation is done stepwise with each single sampling step during the DFT sampling period.

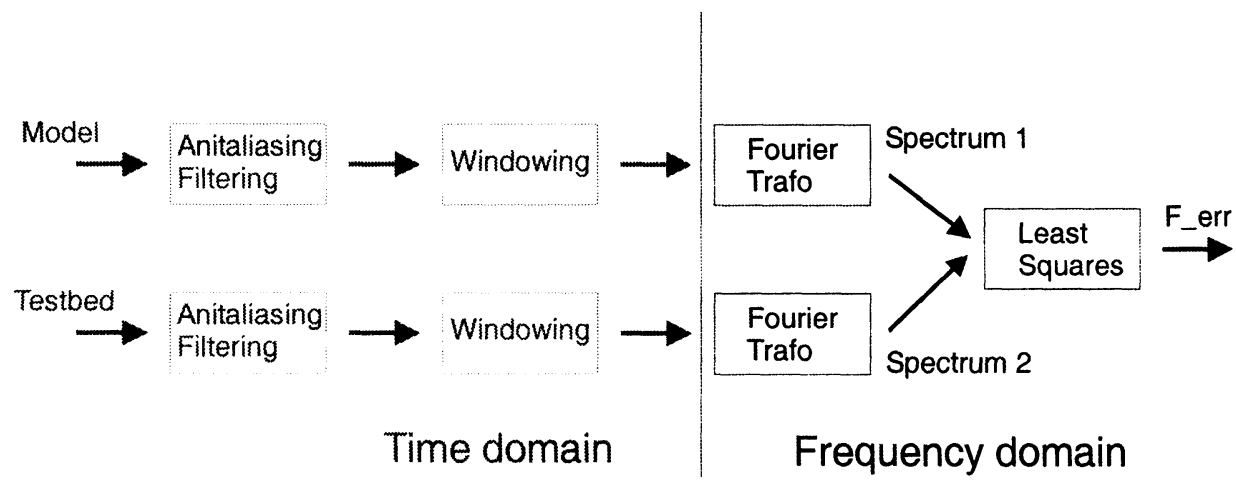

Figure 6. Dataflow of the spectral supervision of the valve slider.

\section{Real-time Aspects}

The phase lag between sampling and computation of a digital realisation has to be reckoned with because it has a huge impact on the controller bandwidth. 
One possibility of modelling the phase lag is to use a dead-time transfer function (Middleton, 1990). It will show that the dead time of a computation has an effect on the phase lag twice as large as that of a sampling. This means that there are two realtime conditions for our digital realisation: firstly, not to overrun the sample limit and secondly, to minimise the time delay between $\mathrm{AD}$ and $\mathrm{DA}$ conversions. Only in the case of a specification for the maximum phase lag can the second condition be described precisely.

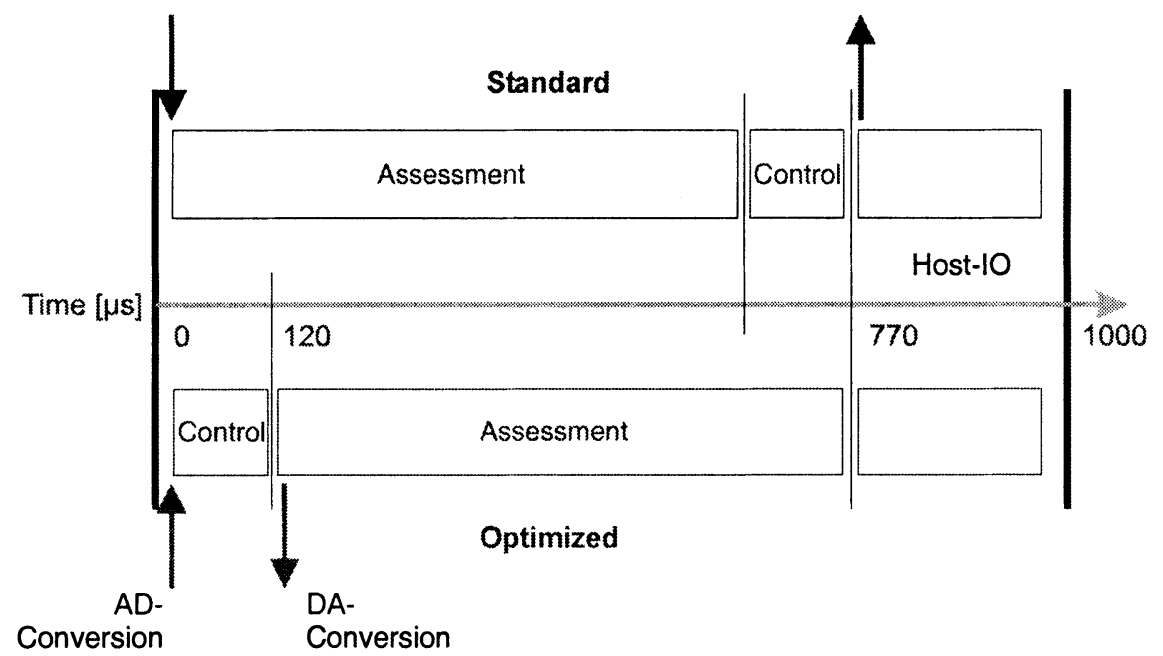

Figure 7. Optimised evaluation to minimise the phase lag of the controller.

Therefore it is important to provide an efficient evaluation order within the sampling period, which is assumed to be $1000 \mu$ s in Figure 7. To minimise the phase lag one has to:

$\square$ delay the effect of the assessment computation to the next sampling period,

start the DA conversion as soon as the required controller output is available.

\section{Real-time Components}

Other recently developed description elements for our modelling tool CAMeL-View are the presented synchronous spectrum analyser, finite-state automata and DA/AD converter elements.

These description elements serve as predefined and validated components to design complex assessment and supervision models.

For real-time realisation we used our simulation platform IPANEMA (Honekamp, 1998) which actually has been ported to run with the $d S P A C E$ Real Time Kernel (Otterbach, Leinfellner, 1999). Figure 8 shows the respective layers. We use the dSPACE DS1005 processor board that is based on the Motorola PowerPC 750 series and the various $D S P A C E$ I/O components. These components are connected to the processor board via the so-called PHS-bus. The DS1005 board is fit for 
multiprocessing tasks, as is our simulation platform IPANEMA. With a very fast optical link called GIGALINK several processor boards can be interconnected:

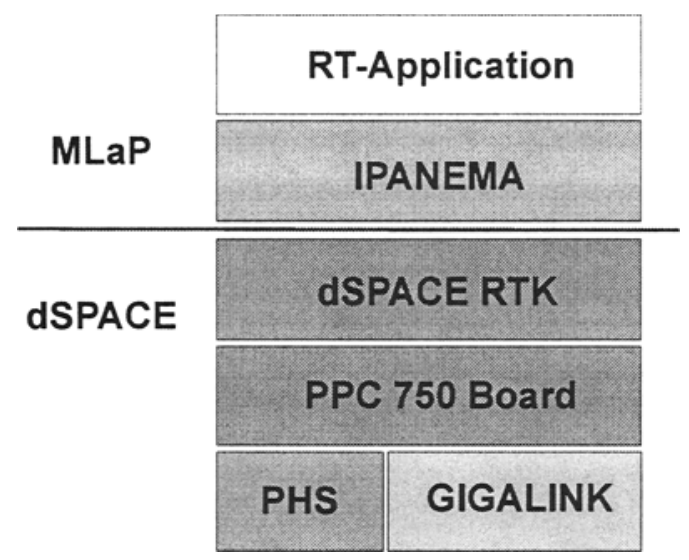

Figure 8. Layers of the real-time hard- and software.

\section{Results}

Examples from the time and the frequency domain were presented to point out our ideas on the supervision and support of online optimisation.

The phase lag problem with digital realisation and our real-time soft- and hardware components were shown in detail. But the most important result is that we have successfully demonstrated our application on the suspension/tilt testbed under hard real-time conditions. The deviation of the cycle time of the application from the average is less than $1 \%$ even during the state switching of the discrete operator parts.

Within the Test Control state of the testbed manual an alteration of controller parameters could result in a higher deviation from the actuator displacement setpoints. Exceeding the limit of $2 \mathrm{~mm}$ results in fact in an automatic switch to the Test Failed state. In this state the robust controller then successfully reduces the deviation to a tolerable value.

Using an inappropriate controller means that the valve sliders could start oscillating at their resonance frequency of about $90 \mathrm{~Hz}$ when excited with a step function. This would lead to a corresponding peak of the auto spectrum and the state of the application in fact would automatically shift to Test Failed. The robust controller then stops this oscillation.

In more general terms, it can be stated that a testbed with a fail-safe layer has been built up to protect the testbed from invalid user operations. This fail-safe behaviour is also of utmost importance for a safe online optimisation. 


\section{Outlook}

The next step will be to implement not only the cost-functions but a proper online optimisation algorithm as a real-time component (Kasper, Lückel, Jäker, Schröer, 1990).

We have seen that some assessments (cost-functions) are extremely expensive. This can be mended by treating them as separate modules to which processors of their own are assigned or which have just a sample rate of their own so that the smallest possible latency for the controllers can be obtained. Therefore the next step will be to implement operator, controller and assessment as pre-emptive multiple tasks with different priorities and sampling rates. Then distributed processing will not be out of reach of the multiple tasks because the simulation platform IPANEMA and even the DS1005 processor board are already laid out for distributed processing.

\section{References}

Honekamp U. (1998). IPANEMA - Verteilte Echtzeit-Informationsverarbeitung in mechatronischen Systemen. Fortschr.-Ber. VDI, Reihe 20, Nr. 267. VDI-Verlag, Düsseldorf, Germany.

Honekamp U., Stolpe R., Naumann R., Lückel J. (1997). Structuring Approach for Complex Mechatronic Systems. $30^{\text {th }}$ International Symposium on Automotive Technology \& Automation - Mechatronics/Automative Electronics, 165-172, Florence, Italy.

Isermann R., Lachmann K.-H., Matko D. (1992). Adaptive Control Systems. Prentice-Hall, New York/London.

Johnson J. R. (1991). Digitale Signalverarbeitung. Hanser, München/Wien, Prentice-Hall, London.

Kasper R, Lückel J., Jäker K.-P., Schröer J. (1990). CACE tool for multi-input, multi-output systems using a new vector optimization method. International Journal of Control, Vol. 51, No. 5, 963-993.

Lückel J., Liu X., Jäker K.-P. (2000). Development of an Active Suspension/Tilt System for a Mechatronic Railway Carriage. $1^{\text {st }}$ IFAC Conference on Mechatronic Systems (Mechatronics 2000), Darmstadt, Germany.

Lückel J., Grotstollen H., Jäker K.-P., Henke M., Liu, X. (1999). Mechatronic Design of a Modular Railway Carriage. 1999 IEEE/ASME International Conference on Advanced Intelligent Mechatronics (AIM'99), Atlanta, GA.

Lückel J., Toepper St., Becker M., Jäker K.-P., Kuhlbusch W., Moritz W. (1999). Innovative Prototypes at the MLaP. International Conference on Engineering Design, ICED 99, Munich, Germany.

Meier-Noe, U., Hahn M. (1999). Entwicklung mechatronischer Systeme mit CAMeL. 3. Workshop Transmechatronik: Entwicklung und Transfer von Entwicklungssystemen der Mechatronik, Krefeld, Germany.

Middleton R. H., Goodwin G. C. (1990). Digital Control and Estimation. Prentice-Hall, Englewood Cliffs, NJ.

Naumann R. (2000). Modellierung und Verarbeitung vernetzter intelligenter mechatronischer Systeme. Fortschr.-Ber. VDI, Reihe 20, Nr. 318. VDI-Verlag, Düsseldorf, Germany.

Otterbach R., Leinfellner R. (1999). Virtuelles Ausprobieren. ELEKTRONIK 8/1999, p. 128134. 